\title{
Répercussions sociales et écologiques de la croissance exponentielle de l'étalement urbain à Montréal
}

\author{
Jaeger $\mathrm{JAG}^{1 *}$, Nazarnia $\mathrm{N}^{1}$
}

\author{
Affiliation \\ ${ }^{1}$ Département de géographie, urbanisme et environnement, Université Concordia, Montréal (Québec) \\ *Correspondance : jochen.jaeger@concordia.ca
}

Citation proposée : Jaeger JAG, Nazarnia N. Répercussions sociales et écologiques de la croissance exponentielle de l'étalement urbain à Montréal. Relevé des maladies transmissibles au Canada 2016;42:231-2. https://doi.org/10.14745/ccdr.v42i10a07f

\section{Contexte}

Les tendances actuelles d'occupation des sols pour créer des zones bâties vont clairement à l'encontre de l'esprit et des principes de la durabilité dans de nombreuses régions du monde. La définition de l'« étalement urbain » n'a pas fini de faire débat, mais la plupart des auteurs s'accordent sur le fait que l'étalement urbain peut être perçu visuellement dans un paysage et que le degré d'étalement est plus élevé lorsque : a) davantage de territoire est construit, b) les bâtiments sont plus dispersés dans le paysage, et c) l'occupation des sols par personne est plus élevée (1).

\section{Objectif}

Faire la synthèse des répercussions sociales et écologiques de l'étalement urbain, expliquer un nouveau moyen de mesurer l'étalement urbain, résumer le degré d'étalement urbain en Suisse (de 1935 à 2010) et à Montréal (de 1951 à 2011) et comparer le degré d'étalement en Europe (de 2006 à 2009) et dans deux villes canadiennes (Montréal et Québec).

\section{Narratif}

L'étalement urbain a beaucoup d'effets écologiques et sociaux, notamment des effets sur la couverture terrestre, le climat local (p. ex. l'effet d'îlot thermique urbain), l'eau, la flore et la faune, ainsi qu'une ségrégation plus marquée en fonction du revenu, un allongement des temps de déplacement et une diminution des interactions sociales (2). L'étalement urbain influe également sur le micro-climat, sur la disponibilité de l'habitat pour les espèces vectrices et hôtes et sur la capacité des vecteurs (3). Par exemple, la croissance, la survie et le comportement des moustiques et des tiques sont très sensibles aux températures dans l'environnement. Dans les climats tempérés, les effets d'îlot thermique urbain peuvent faire augmenter les taux de développement des vecteurs, mais peuvent aussi contrer leur survie et leur comportement alimentaire (3). Les conditions urbaines, telles que l'adéquation de l'habitat et les régimes de température locaux, et l'hétérogénéité des paysages urbains peuvent influer sur les cinq composantes de la capacité des vecteurs : densité des vecteurs, taux de survie, taux de morsure, période $\mathrm{d}^{\prime}$ incubation extrinsèque et compétence des vecteurs. L'étude de l'influence des conditions urbaines sur les processus de transmission constitue une orientation importante pour de futures recherches (3).

Nous avons employé une nouvelle méthode, la prolifération urbaine pondérée, pour quantifier l'étalement urbain et son augmentation en Suisse (depuis 1935, scénarios pour 2050) (1), dans toute l'Europe (de 2006 à 2009) (4) et dans deux villes canadiennes (Montréal et Québec) (5). À Montréal, l'étalement urbain a augmenté de façon exponentielle depuis 1951. À Montréal comme à Québec, l'étalement urbain est devenu un grave problème depuis la fin des années 1980, avec une augmentation à une vitesse sans précédent $(5,6)$.

Sans mesures rigoureuses, l'étalement urbain continuera d'augmenter, mais il existe des cas qui démontrent qu'il est possible de limiter et réduire l'étalement. Par exemple, en Suisse, à l'issue d'une discussion publique intense, la Loi fédérale sur l'aménagement du territoire a été révisée en 2013 pour la rendre plus stricte (1). Les données quantitatives sur l'étalement urbain sont utiles pour surveiller ce qui se passe, pour comparer différents scénarios d'aménagement du territoire et pour établir des cibles et des limites sur le modèle des autres secteurs environnementaux. Par exemple, Hennig et al. (4) ont proposé une stratégie de désétalement pour l'Europe, et la méthode de la prolifération urbaine pondérée a récemment été intégrée au système suisse d'observation du paysage (7). La Banque Alternative Suisse utilise la méthode de la prolifération urbaine pondérée afin d'éviter d'approuver des prêts hypothécaires pour des projets qui contribueraient fortement à l'étalement urbain (8).

\section{Conclusion}

Au cours des 25 dernières années, l'étalement urbain à Montréal a augmenté à une vitesse sans précédent. À Montréal comme à Québec, " on ne parvient plus à maîtriser l'étalement urbain, qui est devenu un problème grave en rapide croissance » (5). L'accélération exponentielle de l'étalement est en contradiction directe avec un développement urbain contrôlé et durable, et 
une meilleure intégration de l'urbanisme et de la planification des transports est nécessaire de toute urgence. Afin d'endiguer l'étalement urbain, le ministère québécois des Transports, de la Mobilité durable et de l'Électrification des transports et la Ville de Montréal doivent travailler main dans la main pour mieux coordonner la politique et la planification. La redistribution du contrôle entre les différents échelons de gouvernement est également en jeu : en effet, les autorités municipales comptent sur l'aménagement du territoire pour maintenir et augmenter leur assiette fiscale, et le gouvernement régional (c'est-à-dire la Communauté métropolitaine de Montréal) n'a pas assez de pouvoir pour limiter l'étalement (9).

On pourrait éviter que cet étalement se poursuive en créant des règlements et des mesures d'incitation adéquats, par exemple en fixant des limites (2). Il peut être utile de tirer des leçons des expériences d'autres pays pour éviter de répéter les erreurs, notamment en réduisant le financement du développement urbain non durable. Pour lutter contre les effets du changement climatique sur les villes, les urbanistes et les habitants de la ville de Montréal travaillent désormais à mieux relier entre eux les espaces naturels de la ville $(6,10)$. Cela pourrait contribuer à réduire l'effet d'îlot thermique urbain et à maintenir la densité des espèces vectrices et hôtes dans des limites raisonnables grâce à l'équilibre dynamique qui se crée dans les communautés écologiques (c'est-à-dire que les relations prédateur-proie des réseaux alimentaires se feraient dans tout un réseau d'espaces naturels plutôt que dans des zones isolées).

\section{Conflit d'intérêts}

Aucun.

\section{Références}

1. Jaeger JAG, Schwick C. Improving the measurement of urban sprawl: Weighted Urban Proliferation (WUP) and its application to Switzerland. Ecol Indic 2014;38:294-308.
2. European Environment Agency (EEA) \& Swiss Federal Office for the Environment (FOEN). Urban sprawl in Europe. Joint EEA-FOEN report. EEA Report 11/2016; ISBN 978-92-9213738-0. Luxembourg: Publications Office of the European Union; 2016. http://www.eea.europa.eu/publications/urbansprawl-in-europe.

3. LaDeau SL, Allan BF, Leisnham PT, Levy MZ. The ecological foundations of transmission potential and vector-borne disease in urban landscapes. Functional Ecology 2015;29: 889901.

4. Hennig El, Schwick C, Soukup T, Orlitová E, Kienast F, Jaeger JAG. Multi-scale analysis of urban sprawl in Europe: towards a European de-sprawling strategy. Land Use Policy 2015;49:48398.

5. Nazarnia N, Schwick C, Jaeger JAG. Accelerated urban sprawl in Montréal, Quebec City, and Zurich: Investigating the differences using time series 1951-2011. Ecol Indic 2016;60:1229-51.

6. Dupras J, Marull J, Parcerisas L, Coll F, Gonzalez A, Girard M et al. The impacts of urban sprawl on ecological connectivity in the Montréal Metropolitan Region. Environ Sci Policy 2016;58:61-73.

7. Kienast F, Frick J, van Strien MJ, Hunziker M. The Swiss Landscape Monitoring Program - a comprehensive indicator set to measure landscape change. Ecol Modell 2015;295:13650 .

8. Alternative Bank of Switzerland (ABS). La Banque Alternative Suisse engage la lutte contre le mitage [communiqué de presse]. 2012 June 18. https://www.abs.ch/fileadmin/ absch/21_Medienmitteilungen/FR/2012_06_18_CP_BAS_ engage_lutte_contre_mitage.pdf.

9. Luka N. Nouveaux enjeux des maladies à transmission vectorielle pour les villes canadiennes. Relevé des maladies transmissibles au Canada 2016;42:241-2. http://www.phacaspc.gc.ca/publicat/ccdr-rmtc/16vol42/dr-rm42-10/ar-12-fra. php.

10. Dupras J, Drouin C, André P, Gonzalez A. Towards the establishment of a green infrastructure in the region of Montréal (Quebec, Canada). Plan Pract Res 2015;30(4):355-75.

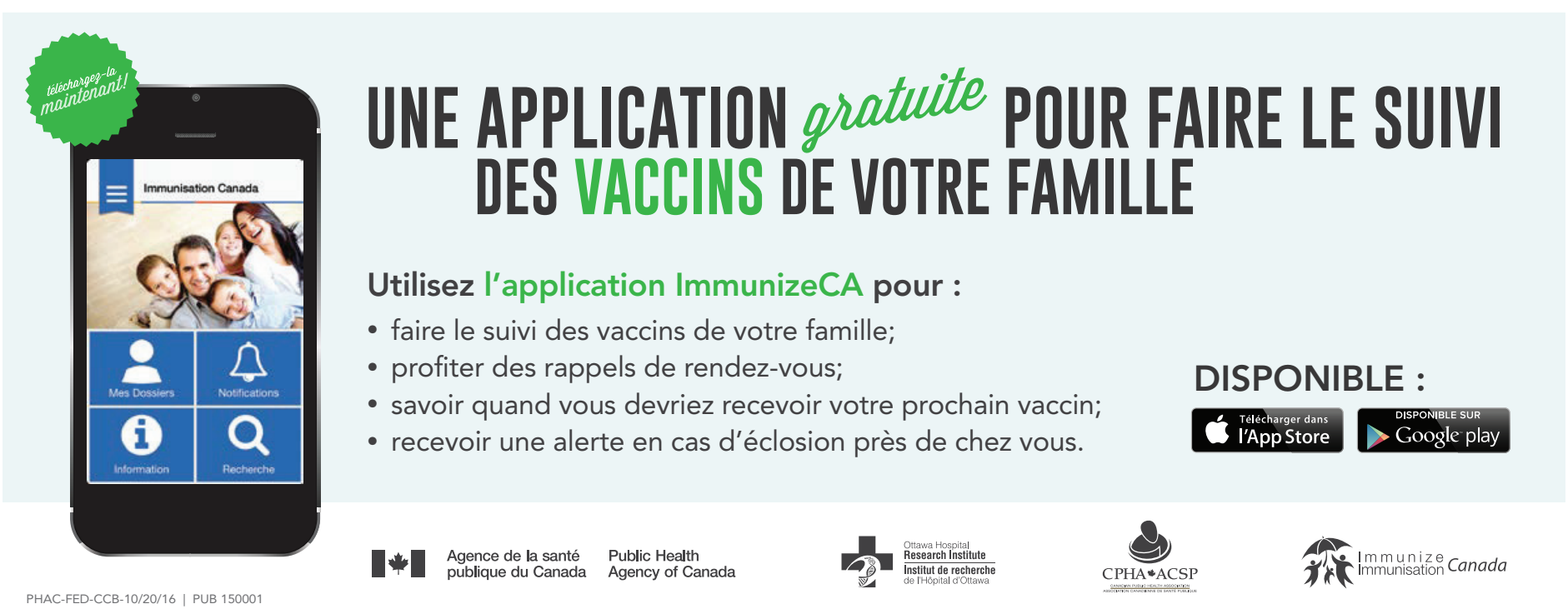

